\title{
Erratum to: IGF-1 deficiency causes atrophic changes associated with upregulation of VGluT1 and downregulation of MEF2 transcription factors in the mouse cochlear nuclei
}

\author{
V. Fuentes-Santamaría · J. C. Alvarado • \\ L. Rodríguez-de la Rosa $\cdot \mathrm{S}$. Murillo-Cuesta $\cdot$ \\ J. Contreras $\cdot$ J. M. Juiz $\cdot$ I. Varela-Nieto
}

Published online: 22 November 2014

(C) Springer-Verlag Berlin Heidelberg 2014

\section{Erratum to: Brain Struct Funct}

\section{DOI 10.1007/s00429-014-0934-2}

Unfortunately, the co-author L. Rodríguez-de la Rosa does not belong to the affiliation referred to in the online published article. The complete and correct author's affiliation list is given here.

The online version of the original article can be found under doi:10.1007/s00429-014-0934-2.

V. Fuentes-Santamaría $(\varangle)$ · J. C. Alvarado · J. M. Juiz Instituto de Investigación en Discapacidades Neurológicas (IDINE), Facultad de Medicina, Universidad de Castilla-La Mancha, Campus de Albacete, C/Almansa 14, 02006 Albacete, Spain

e-mail: veronica.fuentes@uclm.es

L. Rodríguez-de la Rosa $\cdot$ S. Murillo-Cuesta $\cdot$ J. Contreras ·

I. Varela-Nieto

Grupo de Neurobiología de la Audición, Instituto de

Investigaciones Biomédicas Alberto Sols, Consejo Superior de Investigaciones Científicas-Universidad Autónoma de Madrid,

Madrid, Spain

L. Rodríguez-de la Rosa $\cdot$ S. Murillo-Cuesta $\cdot$ J. Contreras ·

I. Varela-Nieto

Centro Investigaciones Biomédicas en Red de Enfermedades

Raras (CIBERER), Instituto de Salud Carlos III, Madrid, Spain

L. Rodríguez-de la Rosa $\cdot$ S. Murillo-Cuesta $\cdot$ J. Contreras ·

I. Varela-Nieto

IdiPAZ Instituto de Investigación en Salud, Madrid, Spain

J. Contreras

Facultad de Veterinaria, Universidad Complutense, Madrid,

Spain 